\title{
Expression of LMP and EBNA genes in Epstein-Barr virus-associated lymphomas in Hu-PBL/SCID mice
}

\author{
YUNLIAN TANG ${ }^{1,2}$, SULI LU ${ }^{1,3}$, XIAONING GAN ${ }^{1}$, FANG LIU $^{1,2}$, YANG ZHANG ${ }^{1,2}$, \\ CHUNYAN LUO ${ }^{1,4}$, YUXIA PAN ${ }^{1}$, LI HONG $^{5}$ and RULIANG GAN ${ }^{1,2}$ \\ ${ }^{1}$ Cancer Research Institute, College of Medicine, University of South China, Hengyang, Hunan 421001; \\ ${ }^{2}$ Key Laboratory of Tumor Cellular and Molecular Pathology, University of South China, Hengyang, \\ Hunan 421001; ${ }^{3}$ Department of Anatomy, Hunan Polytechnic of Environment and Biology, Hengyang, \\ Hunan 421001; ${ }^{4}$ Department of Pathology, Hunan Polytechnic of Environment and Biology, Hengyang, \\ Hunan 421001; ${ }^{5}$ College of Medicine, University of South China, Hengyang, Hunan 421001, P.R. China
}

Received August 6, 2015; Accepted September 26, 2015

DOI: 10.3892/or.2015.4401

\begin{abstract}
Transplantation of peripheral blood lymphocytes (PBLs) from healthy humans with latent Epstein-Barr virus (EBV) infection into severe combined immunodeficiency (SCID) mice results in development of EBV-associated human B-cell lymphoma. However, the expression of EBV genes in relation to lymphoma development has not been reported. We investigated latent membrane protein (LMP) and EBV nuclear antigen (EBNA) gene expression in PBLs from EBV-positive blood donors and induced-lymphoma cells from SCID mice to elucidate the functions and effects of the EBV genome in the occurrence and development of lymphoma. PBLs were isolated from 9 healthy blood donors and transplanted into SCID mice. Gene expression levels of LMP-1, LMP-2A, and LMP-2B and EBNA-1, EBNA-2, EBNA-3A, EBNA-3B, EBNA-3C and EBNA-LP were monitored by real-time quantitative-polymerase chain reaction (qRT-PCR) in cells from nine EBV-induced lymphomas and in matched lymphocytes from healthy subjects. LMP-1, EBNA-1 and EBNA-2 protein levels were detected by western blotting. As a result, LMP-1, LMP-2A and LMP-2B mRNA levels were upregulated 256-, 38- and 331-fold, respectively, in the EBV-induced lymphoma cells compared with the controls, while EBNA-1 and EBNA-3A mRNA levels were upregulated 1157- and 1154-fold, respectively. EBNA-2, EBNA-3B, EBNA-3C and EBNA-LP mRNAs were detected in lymphoma cells, but not in lymphocytes from EBV-positive blood donors. LMP-1 and EBNA-2 proteins were
\end{abstract}

Correspondence to: Professor Li Hong or Professor Runliang Gan, Cancer Research Institute, College of Medicine, University of South China, 28 Chang Sheng Xi Avenue, Hengyang, Hunan 421001, P.R. China

E-mail: hongli1230@126.com

E-mail: gan998@yahoo.com

Key words: Epstein-Barr virus, induced lymphoma, viral gene expression, latent membrane protein, EBV nuclear antigen, SCID mouse not expressed in lymphocytes from EBV-positive blood donors, according to western blotting. Weak EBNA-1 expression was observed in lymphocytes from blood donors with latent EBV infection, while LMP-1, EBNA-1 and EBNA-2 protein levels were significantly upregulated in EBV-induced lymphoma cells, consistent with mRNA expression levels detected by qRT-PCR. In conclusion, LMP-1, LMP-2A, LMP-2B, EBNA-1 and EBNA-3A were upregulated in EBV-induced lymphoma cells, while EBNA-2, EBNA-3B, EBNA-3C and EBNA-LP were absent in lymphocytes from humans with latent EBV infection, but were positively expressed in EBV-induced lymphoma cells.

\section{Introduction}

Epstein-Barr virus (EBV) is a human lymphotrophic double-stranded $\gamma$-DNA herpes virus. EBV infection is common within the global population, with $\sim 90 \%$ of adults testing positive for serum viral capsid antigen (VCA)-IgG antibody. Latent EBV usually persists after infection, and may lead to malignant transformation of host cells. EBV has been found to be involved in the occurrence and progression of nasopharyngeal carcinoma, lymphoma, gastric cancer, and other malignancies, and is considered as a human tumor virus (1). EBV infection of resting B lymphocytes in vitro may induce cell activation and proliferation, potentially resulting in the establishment of immortalized lymphoblastoid cell lines (LCLs). These LCLs express virally-encoded proteins, including $6 \mathrm{EBV}$ nuclear proteins (EBNAs), EBNA-1, -2, -3A, $-3 \mathrm{~B},-3 \mathrm{C}$ and $-\mathrm{LP}$, and 3 latent membrane proteins (LMPs), LMP-1, -2A and -2B (2,3), associated with cell proliferation and tumorigenesis.

We previously confirmed that transplantation of peripheral blood lymphocytes (PBLs) from healthy human subjects with latent EBV infection into severe combined immunodeficiency (SCID) mice induced EBV-associated human-origin B-cell lymphoma $(4,5)$. However, there has been no comprehensive and systematic evaluation of viral LMP and EBNA gene products in EBV-associated lymphomas. In the present study, 
we evaluated EBV gene and protein expression levels in EBV-induced lymphoma cells in SCID mice using quantitative real-time polymerase chain reaction (qRT-PCR) and western blotting, and compared them with matched lymphocytes from healthy human blood donors with latent EBV infection to reveal the changes in EBV gene expression profiles associated with EBV-induced lymphoma.

\section{Materials and methods}

Materials. Peripheral venous blood (300-400 ml) from nine healthy blood donors was provided by Hengyang Blood Center. SCID-Beige mice were purchased from Beijing Weitong Lihua Experimental Animal Technology Co., Ltd. (Beijing, China). The present study protocol was approved by the Medical Ethics Committee of the University of South China.

\section{Methods}

Detection of the EBV infection status of blood donors. Plasma EBV VCA-IgG was detected using an EBV-VCA-IgG ELISA kit (ADL Embedded Solutions Inc., San Diego, CA, USA). The presence of EBV DNA in the donor blood cells was detected by extraction of DNA from whole blood, followed by PCR amplification of the 82-bp LMP-1 gene sequence (GI: 896226, forward, 5'-CTGCTCATCGCTCTCTGGAA-3' and reverse, 5'-AGACAAGTAAGCACCCGAAGATG-3') (2,3). The PCR reaction conditions were as follows: a 4-min pre-denaturation at $94^{\circ} \mathrm{C}$, a 30 -sec denaturation at $94^{\circ} \mathrm{C}$, a $30-\mathrm{sec}$ annealing at $52^{\circ} \mathrm{C}$, a 30 -sec extension at $72^{\circ} \mathrm{C}$, for 30 cycles, followed by a 5 -min extension at $72^{\circ} \mathrm{C}$. The size of the amplified product was $82 \mathrm{bp}$. The PCR products were identified using $2 \%$ agarose gel electrophoresis, with LMP-1 amplification results observed under UV using a gel-imaging and analysis system.

Construction of Hu-PBL/SCID chimeric mice. Hu-PBL/SCID chimeric mice were constructed by inoculation of SCID mice with lymphocytes isolated from the peripheral blood of human donors with latent EBV infection. PBLs were isolated using lymphocyte separation medium (Tianjin Hao Yang Biological Manufacture Co., Ltd., Tianjin, China) from peripheral venous blood of healthy adults with latent EBV infection, diluted to $8-10 \times 10^{7} / \mathrm{ml}$ using RPMI-1640 culture medium without fetal bovine serum. Each SCID mouse was inoculated intraperitoneally with $1 \mathrm{ml}$ PBL suspension under aseptic conditions. PBLs from each blood donor were used to inoculate 3 or 4 SCID mice. After inoculation, each SCID mouse was administered cyclosporin A (Sandoz Co., Novartis, Switzerland) via intraperitoneal injection at $10 \mathrm{mg} / \mathrm{kg} /$ day for 2 consecutive days. The dose was adjusted to $15 \mathrm{mg} / \mathrm{kg}$ every other day from the third day, for a total of 11 doses.

Development and pathological examination of EBV-induced tumors in SCID mice. Hu-PBL/SCID chimeric mice were maintained in a laminar-air-flow rack under specific pathogen-free conditions. The mice were euthanized to reduce suffering in the event of death or sickness, and surviving mice were sacrificed 4 months after PBL inoculation. All mice were subjected to detailed autopsy. The abdominal and mediastinal cavities and vital organs were examined, and tumor shape, size, color, texture, and invasion of adjacent organs were examined. Each induced tumor was divided into two parts: one part was frozen in liquid nitrogen for RNA, DNA, or protein extraction; the other part was fixed in $10 \%$ neutral formalin, embedded in paraffin, and sliced into $4-\mu \mathrm{m}$ serial sections for routine hematoxylin and eosin or immunohistochemical staining.

Immunohistochemical staining was performed using antibodies against human leukocyte common antigen CD45 (LCA), B-cell markers (CD20 and CD79a), and T-cell markers (CD45RO and CD3) (Maixin Biotech. Co., Ltd., Fuzhou, China). The SP immunohistochemistry and DAB kits were purchased from Fuzhou Maixin Biotechnology Co., Ltd. (China). Staining was carried out according to the manufacturer's instructions. Phosphate-buffered saline replaced the primary antibody as a negative control.

PCR detection of human-specific Alu sequence in induced tumors in SCID mice. DNA was extracted from tumors induced in SCID mice and the 221-bp human-specific Alu sequence was amplified using PCR (5). The sequence of the human Alu primer was as follows: forward, 5'-CACCTGTAA TCCCAGCAGTTT-3' and reverse, 5'-CGCGATCTCGGCTC ACTGCA-3'. The PCR product was identified by $1.5 \%$ agarose gel electrophoresis and observed under UV light using a gel-imaging analysis system.

Determination of EBV gene expression in induced tumors by $q R T-P C R$. Total RNA was extracted and purified and used to synthesize cDNA. An RNA extraction kit (Omega Bio-Tek Inc., Doraville, GA, USA) was used to extract total RNA from induced tumor tissues and matched lymphocytes from 'normal' donors, with $\mathrm{B}_{95-8}$ cells as a positive control ( $\mathrm{B}_{95-8}$ cells were derived from marmoset leukocytes transformed by EBV). The quality and quantity of the RNA samples were determined using a UV spectrophotometer (Perkin-Elmer, Fremont, CA, USA), followed by storage at $-80^{\circ} \mathrm{C}$. In accordance with the instructions of the reverse transcription kit (Promega Corporation, Madison, WI, USA), $2 \mu \mathrm{g}$ RNA from each sample was used for cDNA synthesis. mRNA expression was detected by qRT-PCR using SYBR Premix Ex Taq reagent (Takara, Dalian, China). qRT-PCR was carried out in a 96-well plate using an ABI 7700 Real-Time PCR system (Applied Biosystems, Foster City, CA, USA). The primers used are listed in Table I.

RNA from $\mathrm{B}_{95-8}$ cells was used as a template to obtain cDNA for qRT-PCR. A standard curve was established based on the initial cDNA duplication number and CT value for real-time fluorescent quantitative detection of $\mathrm{B}_{95-8}$ cells. The template content in the sample was determined by relative quantitation using the $\Delta \Delta C T$ method, standardized by the duplication number of the housekeeping gene glyceraldehyde 3-phosphate dehydrogenase. Each sample was tested in triplicate.

Determination of EBV protein expression in the induced tumors by western blotting. Total protein was extracted from sodium dodecyl sulfate (SDS) lysates (SDS lysis buffer; Beyotime, China) of induced tumor tissues and matched 'normal' lymphocytes. Proteins were quantified using a BCA kit (Enhanced BCA protein assay kit; Beyotime). Protein samples $(50 \mu \mathrm{g})$ were separated using $8-12 \%$ SDS-polyacrylamide gel 
Table I. Primer sequences of qRT-PCR (5'-3').

\begin{tabular}{lllr}
\hline Gene name & \multicolumn{1}{c}{ Forward primer } & \multicolumn{1}{c}{ Reverse primer } & Size (bp) \\
\hline LMP-1 & CTGCTCATCGCTCTCTGGAA & AGACAAGTAAGCACCCGAAGATG & 82 \\
LMP-2A & CGTCACTCGGACTATCAACCAC & CTTCCTCTGCCCGCTTCTT & 149 \\
LMP-2B & CGCCGTTTGACTGTTTGTG & AGCAGCAGCGTCATGGAA & 125 \\
EBNA-1 & GTTCCTCGCCTTAGGTTGTA & AGCTCTCCTGGCTAGGAGTC & 124 \\
EBNA-2 & GTCTGGCACATGCAAGACA & TCTGCCACCTGCAACACTAA & 154 \\
EBNA-3A & CTAATGGCCTGTCGAATGG & TTTCAGCGCATCGACACA & 103 \\
EBNA-3B & GGATCGTCACCACCATTGT & GGTGGGATCTGAGCCTATTT & 159 \\
EBNA-3C & GGCACATTGTCTTCCGTGTC & TACAGACTACCGGCGAGCAT & 138 \\
EBNA-LP & TCCCCTCGGACAGCTCCTA & CCACTTACCACCTCCCCTTCT & TGGTGAAGACGCCAGTGGA \\
GAPDH & GCACCGTCAAGGCTGAGAAC & TGA &
\end{tabular}

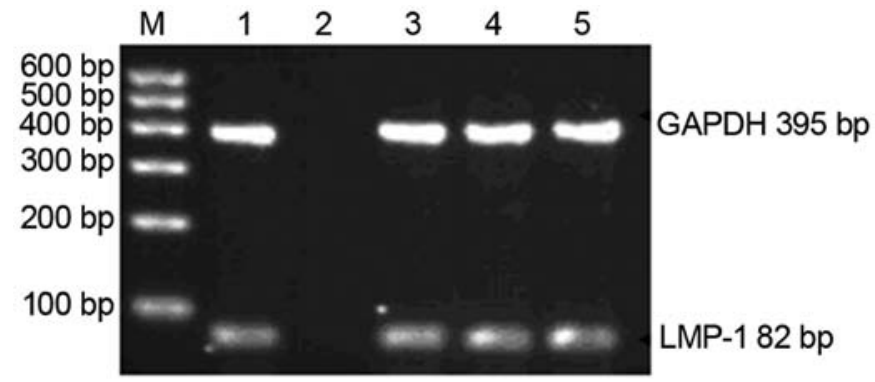

Figure 1. Agarose gel electrophoresis of the PCR amplification products of the LMP-1 gene ( $82 \mathrm{bp}$ ). Lane M, marker; lane 1, positive control ( $\mathrm{B}_{95-8}$ cells); lane $2, \mathrm{ddH}_{2} \mathrm{O}$ negative control; lanes $3,4,5$, whole blood cells from blood donors.

electrophoresis and electrotransferred to polyvinylidene difluoride membranes (Millipore Corporation, Boston, MA, USA). Membranes were blocked in Tris-buffered saline containing $5 \%$ skimmed milk (TBST; $25 \mathrm{~mm}$ Tris- $\mathrm{HCl}, 150 \mathrm{mM} \mathrm{NaCl}$, $\mathrm{pH} 7.5$ and $0.05 \%$ Tween-20) for $4 \mathrm{~h}$, followed by the addition of the following diluted primary antibodies containing $5 \%$ skimmed milk: EBNA-1 (mouse monoclonal anti-EBNA-1; Santa Cruz Biotechnology, Santa Cruz, CA, USA), EBNA-2 (rat monoclonal anti-EBNA-2; Millipore Corporation) and LMP-1 (mouse monoclonal anti-EBV LMP-1; Dako, Glostrup, Denmark), with rat anti-human $\beta$-actin (anti- $\beta$-actin mouse monoclonal antibody; Beijing ComWin Biotech, China) as an internal control, at $4{ }^{\circ} \mathrm{C}$ overnight. The TBST membrane was rinsed four times for $10 \mathrm{~min}$ each. The corresponding secondary antibodies were then added (Beijing ComWin Biotech), followed by incubation for $2 \mathrm{~h}$ at room temperature. The membrane was then rinsed another four times for 10 min each. ECL Plus luminescence reagent (Pierce ECL Western Blotting Substrate; Pierce, Rockford, IL, USA) was then dripped onto the membrane, followed by pressing, image developing, and fixing in an X-ray machine, and image collection using a fluorescence image-analysis system. Each sample was tested in triplicate.

Statistical analysis. All experimental data are presented as mean \pm standard deviation. Induced lymphoma cells and matched 'normal' lymphocytes were compared by t-tests, using SPSS13.0 software. A value of $\mathrm{P}<0.05$ was considered to indicate statistical significance.

\section{Results}

EBV-infection status of the blood donors. All 9 blood donors tested positive for latent EBV infection according to both EBV-VCA-IgG detection and PCR amplification of the LMP-1 gene of EB virus (Fig. 1).

Tumorigenesis in the SCID mice. Tumors were formed in the mediastinum and intraperitoneal cavity of the SCID mice. The induced tumors appeared nodular, gray, or gray-red to the naked eye (Fig. 2A), with a fish-like texture in cross-section. Under light microscopy, the tumor cells demonstrated mixed morphology resembling plasmacytoid lymphocytes, centroblastic cells and immunoblasts (Fig. 2B). Immunohistochemical staining of the induced tumors was negative for T-cell markers (CD3 and CD45RO) (Fig. 2C) and positive for LCA B-cell markers (CD20 and CD79a) (Fig. 2D), and consistent with the pathological diagnosis of diffuse large B-cell lymphoma.

Determination of the induced tumor origin using Alu PCR. DNA was extracted from the tumor tissues, followed by PCR amplification of the 221-bp human-specific Alu sequence, which confirmed that the induced lymphomas were human in origin (Fig. 3).

LMP and EBNA mRNA expression in induced lymphomas. mRNA expression levels of the three LMPs were detected in induced-lymphoma cells from nine $\mathrm{Hu}-\mathrm{PBL} / \mathrm{SCID}$ chimeric mice and matched PBLs from donors by fluorescence quantitative PCR (Table II). LMP-1 gene expression in the induced-lymphoma cells was increased 256-fold compared with the expression level in the 'normal' lymphocytes $(\mathrm{P}<0.05)$, LMP-2A was upregulated 38-fold $(\mathrm{P}<0.05)$, and LMP-2B was upregulated 331-fold $(\mathrm{P}<0.05)$.

mRNA expression levels of the six EBNA genes were also detected by fluorescence quantitative PCR in induced-lymphoma cells and matched PBLs (Table III). EBNA-1 expression in the induced-lymphoma cells was upregulated 1,157-fold compared with the expression level 

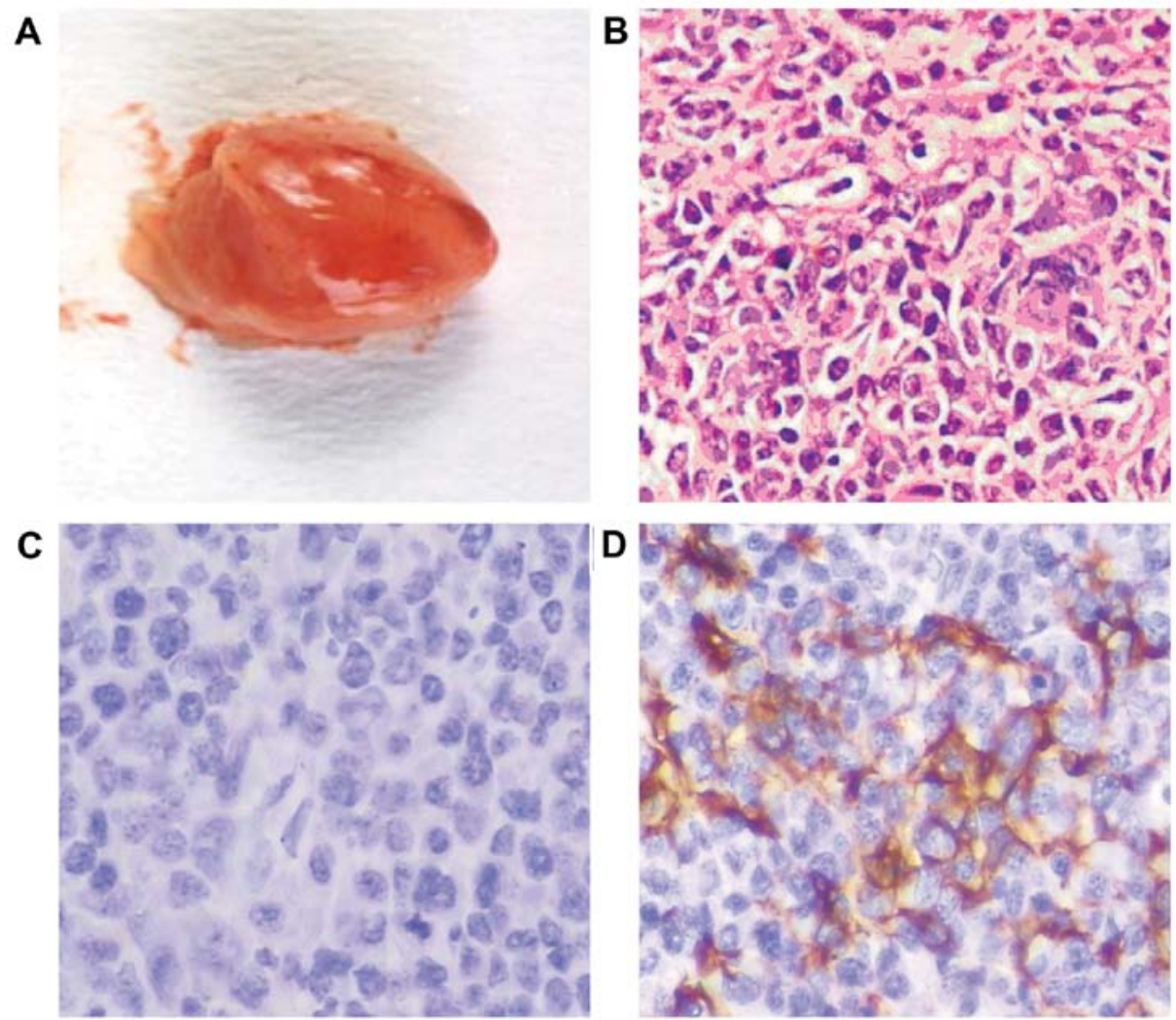

Figure 2. Pathological examinations of induced tumors in the SCID mice. (A) Tumors appeared gray or gray-red. (B) Under light microscopy, the cells demonstrated mixed morphology including plasmacytoid lymphocytes, cells with small cleaved nuclei, large cleaved nuclei, small and large cells without cleaved nuclei, and immunoblasts. (C) Cells tested negative for T-cell markers (CD3); (D) and positive for B-cell markers (CD20).

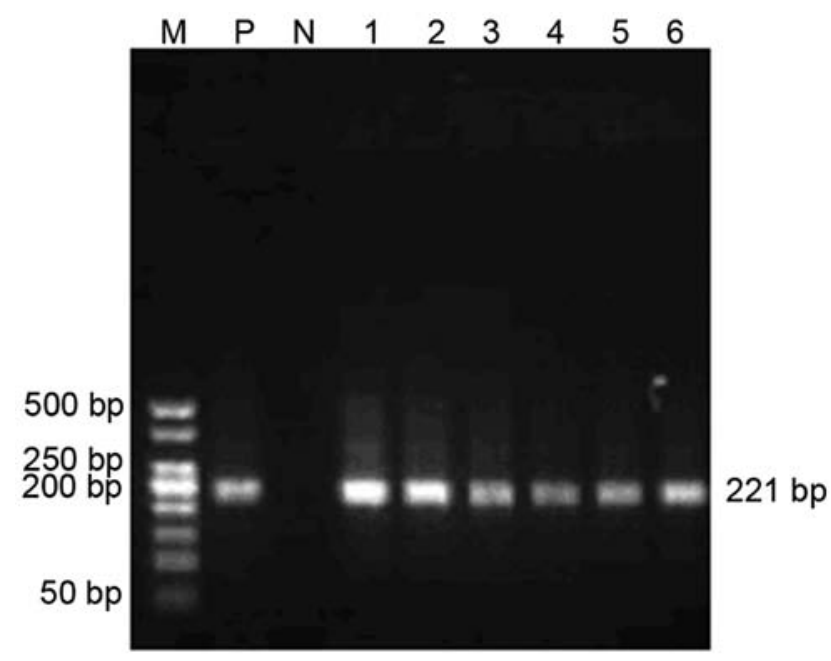

Figure 3. PCR amplification of Alu sequence (221 bp) by agarose gel electrophoresis. Lane M, marker; lane P, positive control (human lymphocytes); lane N, negative control (mouse liver tissue); lanes 1-6, induced lymphoma in Hu-PBL/SCID chimeric mice.

in the 'normal' lymphocytes $(\mathrm{P}<0.05)$, and EBNA-3A was upregulated 1,154-fold $(\mathrm{P}<0.05)$. EBNA-2, EBNA-3B, EBNA-3C and EBNA-LP were not expressed in the 'normal' lymphocytes, but were significantly expressed in the inducedlymphoma cells.

Detection of LMP-1, EBNA-1 and EBNA-2 protein expression by western blotting. LMP-1, EBNA-1 and EBNA-2
Table II. Expression of LMPs in the EBV-induced lymphoma cells and normal human lymphocytes (relative mRNA).

\begin{tabular}{lcll}
\hline & $\begin{array}{c}\text { Normal } \\
\text { lymphocytes } \\
\left(\text { mean } \pm \text { SD) } \times 10^{-4}\right.\end{array}$ & $\begin{array}{c}\text { EBV-induced } \\
\text { lymphoma } \\
\left(\text { mean } \pm \text { SD) } \times 10^{-4}\right.\end{array}$ & P-value \\
\hline LMP-1 & $2.414 \pm 1.080$ & $617.402 \pm 122.101$ & $<0.05$ \\
LMP-2A & $2.975 \pm 1.640$ & $112.101 \pm 8.382$ & $<0.05$ \\
LMP-2B & $0.640 \pm 0.304$ & $211.603 \pm 48.420$ & $<0.05$ \\
\hline
\end{tabular}

Note: Comparison between EBV-induced lymphoma cells and normal lymphocytes $(n=9)$.

protein expression levels in the EBV-induced lymphoma cells and matched 'normal' lymphocytes were detected by western blotting. LMP-1, EBNA-1 and EBNA-2 protein levels were significantly upregulated in the EBV-induced lymphomas (Fig. 4), consistent with the mRNA expression trends demonstrated by fluorescence quantitative PCR. The EBNA-2 protein level was also increased in the EBV-induced lymphoma cells compared with that in the matched 'normal' lymphocytes before transplantation.

\section{Discussion}

EBV is an important human tumor virus, the oncogenic effect of which is mainly realized through viral gene transcription 
Table III. Expression of EBNAs in the EBV-induced lymphoma cells and normal human lymphocytes (relative mRNA).

\begin{tabular}{lccc}
\hline & $\begin{array}{c}\text { Normal } \\
\text { lymphocytes } \\
\text { (mean } \pm \text { SD) } 10^{-4}\end{array}$ & $\begin{array}{c}\text { EBV-induced } \\
\text { lymphoma }\end{array}$ & \\
(mean \pm SD) $10^{-4}$ & P-value \\
\hline EBNA-1 & $0.318 \pm 0.034$ & $368.041 \pm 33.502$ & $<0.05$ \\
EBNA-2 & $0.000 \pm 0.011$ & $56.680 \pm 19.867$ & $<0.05$ \\
EBNA-3A & $0.324 \pm 0.046$ & $374.033 \pm 116.041$ & $<0.05$ \\
EBNA-3B & $0.000 \pm 0.012$ & $248.107 \pm 193.501$ & $<0.05$ \\
EBNA-3C & $0.000 \pm 0.007$ & $339.413 \pm 195.020$ & $<0.05$ \\
EBNA-LP & $0.000 \pm 0.003$ & $322.105 \pm 84.801$ & $<0.05$ \\
\hline
\end{tabular}

Note: Comparison between EBV-induced lymphoma cells and normal lymphocytes $(n=9)$.

and the effects of the encoded proteins on the biological behavior of the infected cells. It is therefore necessary to understand the process of EBV genome transcription and expression during tumorigenesis, as well as its functional activity in its host cells (6-8).

LMP-1 has been considered to be the most important oncogenic EBV gene, able to mediate cell proliferation and inhibit apoptosis. Using qRT-PCR and western blotting, we showed that LMP-1 expression was upregulated in the EBV-induced lymphoma cells compared with the expression level in the original 'normal' lymphocytes with latent EBV infection, confirming and supporting the role of LMP-1 in EBV-associated lymphomas. Zhang et al constructed transgenic mice expressing LMP-1 and confirmed its important role in B-cell proliferation and transformation (9). Increased expression of LMP-1 protein promoted cell proliferation in NK/T cell lymphoma (10). Previous studies also showed that LMP-1 activated $\beta$-catenin through the phosphatidylinositol 3-kinase/Akt signaling pathway, associated with the proliferation of EBV-infected B cells (11). LMP-1 could regulate DAPK1 expression and activate NF- $\kappa B$ signaling in LCLs (12). Transcription products of LMP-2A could be sustainably detected in EBV-associated malignant tumors, suggesting that LMP-2A may play an important role in persistent in vivo viral infection and EBV-associated diseases (13). LMP-2A gene expression was upregulated 38-fold in EBV-induced lymphoma cells compared with 'normal' lymphocytes, according to qRT-PCR $(\mathrm{P}<0.05)$, while LMP-2B was upregulated 331-fold $(\mathrm{P}<0.05)$. These results suggest that LMP-2A and LMP-2B are also involved in the occurrence and development of EBV-induced lymphomas. Previous studies reported that LMP-2A could promote the malignant transformation of cells and the survival and activation of $\mathrm{B}$ cells by increasing the expression of genes associated with cell cycle induction and apoptosis inhibition, together with LMP-1 $(14,15)$. Through regulating tumor necrosis factor receptor-associated factor 2 expression, LMP-2A regulates NF- $\kappa \mathrm{B}$ signaling pathway activation mediated by LMP-1 $(16,17)$, thus helping lymphoma cells to escape apoptosis. LMP-2B regulates the function of LMP-2A (13), preventing the potential lysis of EBV, while high
1 2

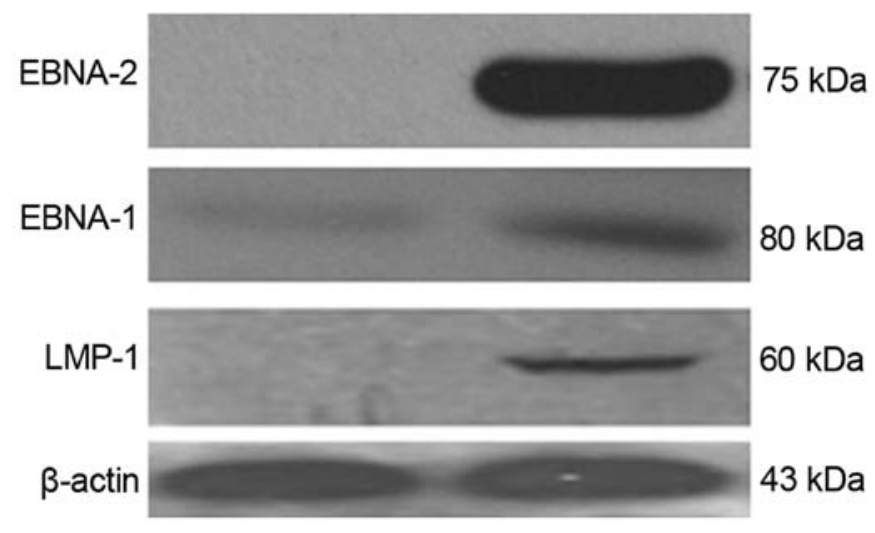

Figure 4. Determination of LMP-1, EBNA-1 and EBNA-2 protein expression levels by western blotting. LMP-1 and EBNA-2 were not expressed in 'normal' human lymphocytes with latent EBV infection, while weak EBNA-1 expression was observed. LMP-1, EBNA-1 and EBNA-2 protein levels were significantly increased in EBV-induced lymphoma cells. Lane 1, matched 'normal' lymphocytes; lane 2, EBV-induced lymphoma cells.

levels of LMP-2B expression also accelerate the transition from latent to proliferative EBV infection.

EBNA-1 appears to be expressed in all EBV-associated tumors, with significant implications for the stability of the EBV genome in the host cells (18), which is in turn crucial for the maintenance, replication, and transcription of the EBV episome (19). EBNA-1, LMP-1 and LMP-2A may participate in the progression of a variety of human malignant tumors induced by EBV, and expression of these genes in the infected cells thus indicates a potential risk of cancer (20). Our qRT-PCR and western blotting results showed that EBNA-1 was upregulated in EBV-induced lymphoma cells compared with the level in the 'normal' lymphocytes. In addition to helping to maintain the viral episome, EBNA-1 can also control viral replication and gene expression. Acting via a hidden cis-acting mechanism, EBNA1 can help virus-carrying host cells to escape the host immune system (21).

EBNA-2 is one of first viral gene products to be expressed in host cells after EBV infection and plays a key role in the immortalization of infected B cells (22). EBNA-2 is directly responsible for initiating the transcription of EBV-related proteins during the type III incubation period, resulting in excessive growth of LCLs (23). After binding to CBF1/RBP-JK, EBNA-2 promotes the expression of EBNA genes and LMP-1 protein, thus promoting cell growth and proliferation (24). EBNA-2 can also activate the signal transduction pathway mediated by JAK-STAT, leading to continuous proliferation of lymphocytes (25). The present study demonstrated high levels of EBNA-2 expression in induced-lymphoma cells, suggesting that it may play an important role in the pathogenesis of EBV-associated lymphomas.

EBNA-3 consists of three subtypes EBNA-3A, EBNA-3B and EBNA-3C associated with gene regulation. Young et al (26) found that EBNA-3A induced the re-distribution of heat shock protein 70 in immortalized EBV-induced B lymphocytes in vitro, promoting the expression of chaperone and co-chaperone proteins in the host cells. Although EBNA-3B is not essential for B-cell immortalization, its expression 
plays an important role in the proliferation and initiation of EBV-infected B lymphocytes (27). No previous studies have reported on the expression of EBNA-3 and EBNA-LP genes in tumor cells, and their roles and functions remain unknown. However, the present study found that EBNA-3A gene expression was upregulated in EBV-induced tumor cells compared with this level in the 'normal' lymphocytes. EBNA-3B, EBNA-3C and EBNA-LP genes were not expressed in 'normal' cells, but were positively expressed in EBV-induced lymphoma cells. Further studies are needed to clarify the roles of EBNA-3 and EBNA-LP. Tursiella et al (28) inhibited EBNA-3A expression by transfection of EBNA-3A-specific shRNA and observed that the cell cycle in Wp-R BL Sal cells and LCLs stopped at $G_{0} / G_{1}$, with growth inhibition and increased apoptosis, together with upregulation of $\mathrm{p} 21^{\mathrm{WAF} 1 / \mathrm{CIP} 1}$ and Bim expression. Wp-R BL Sal cells may suppress the expression of Bim through EBNA-3A, inhibiting Bim-mediated apoptosis and thus affecting the oncogenic potential. EBNA-3C is one of the essential antigens required for in vitro primary B-cell transformation. EBNA-3C acts as a transcriptional co-regulator by interacting with various cellular and viral factors (29). EBNA-LP and EBNA-2 were found to be co-expressed in EBV-infected B lymphocytes and to play a critical role in the survival and growth of lymphoblastoids (30). EBNA-LP is also a co-activator of EBNA-2, which can enhance B-cell transformation mediated by EBNA-2 (31). Furthermore, EBNA-LP and EBNA-2 can induce the activation of cellular and viral genes, including LMP-1 and cyclin D2 (32). The above results suggest that EBNA-3 and EBNA-LP may be important oncogenic factors involved in the malignant transformation of lymphocytes by EBV.

The results of the present study showed that LMP and EBNA gene expression levels were upregulated in an EBV-associated lymphoma model, suggesting that their products may play important roles in the malignant transformation of lymphocytes. Further studies are needed to explore the mechanisms responsible for the actions of these EBV genes.

\section{Acknowledgements}

This research was supported by the National Natural Science Foundation of China (grant nos. 81272182 and 81372134), and the Construct Program of the Key Discipline in Hunan Province (2011-76), and Hunan Province Cooperative Innovation Center for Molecular Target New Drug Study (2014-405).

\section{References}

1. Lan K, Verma SC, Murakami M, Bajaj B and Robertson ES: Epstein-Barr virus (EBV): Infection, propagation, quantitation, and storage. Curr Protoc Microbiol Chapter 14: Unit 14E.2, 2007. doi: 10.1002/9780471729259.mc14e02s6.

2. Rasul AE, Nagy N, Sohlberg E, Ádori M, Claesson HE, Klein G and Klein E: Simultaneous detection of the two main proliferation driving EBV encoded proteins, EBNA-2 and LMP-1 in single B cells. J Immunol Methods 385: 60-70, 2012.

3. Tang Y, Luo C, Cheng A, Lu S, Xu J, Fu T and Gan R: Expression of latent membrane proteins in Epstein Barr virus-transformed lymphocytes in vitro. Mol Med Rep 10: 1117-1121, 2014.

4. Gan R, Yin Z, Liu T, Wang L, Tang Y and Song Y: Cyclosporine A effectively inhibits graft-versus-host disease during development of Epstein-Barr virus-infected human B cell lymphoma in SCID mouse. Cancer Sci 94: 796-801, 2003.
5. Gan R, Xie X, He J, Liu X, Hong L, Tang Y, Liu F and Xie H: Gene analysis of Epstein-Barr virus-associated lymphomas in $\mathrm{Hu}-\mathrm{Pbl} / \mathrm{SCID}$ chimeras. Tumori 96: 465-472, 2010.

6. Klein E, Kis LL and Klein G: Epstein-Barr virus infection in humans: From harmless to life endangering virus-lymphocyte interactions. Oncogene 26: 1297-1305, 2007.

7. Canaan A, Haviv I, Urban AE, Schulz VP, Hartman S, Zhang Z, Palejev D, Deisseroth AB, Lacy J, Snyder M, et al: EBNA1 regulates cellular gene expression by binding cellular promoters. Proc Natl Acad Sci USA 106: 22421-22426, 2009.

8. Klein G, Klein E and Kashuba E: Interaction of Epstein-Barr virus (EBV) with human B-lymphocytes. Biochem Biophys Res Commun 396: 67-73, 2010.

9. Zhang B, Kracker S, Yasuda T, Casola S, Vanneman M, Hömig-Hölzel C, Wang Z, Derudder E, Li S, Chakraborty T, et al: Immune surveillance and therapy of lymphomas driven by Epstein-Barr virus protein LMP1 in a mouse model. Cell 148: 739-751, 2012.

10. Ramakrishnan R, Donahue H, Garcia D, Tan J, Shimizu N, Rice AP and Ling PD: Epstein-Barr virus BART9 miRNA modulates LMP1 levels and affects growth rate of nasal NK T cell lymphomas. PLoS One 6: e27271, 2011.

11. Tomita M, Dewan MZ, Yamamoto N, Kikuchi A and Mori N: Epstein-Barr virus-encoded latent membrane protein 1 activates beta-catenin signaling in B lymphocytes. Cancer Sci 100: 807-812, 2009.

12. Lee CW, Leu SJ, Tzeng RY, Wang SF, Tsai SC, Sun KH, Chen RH and Huang JC: Latent membrane protein 1 of Epstein-Barr virus regulates death-associated protein kinase 1 in lymphoblastoid cell line. Virology 413: 19-25, 2011.

13. Rechsteiner MP, Berger C, Zauner L, Sigrist JA, Weber M, Longnecker R, Bernasconi M and Nadal D: Latent membrane protein $2 \mathrm{~B}$ regulates susceptibility to induction of lytic Epstein-Barr virus infection. J Virol 82: 1739-1747, 2008.

14. Bultema R, Longnecker R and Swanson-Mungerson $M$ : Epstein-Barr virus LMP2A accelerates MYC-induced lymphomagenesis. Oncogene 28: 1471-1476, 2009.

15. Wasil LR, Tomaszewski MJ, Hoji A and Rowe DT: The effect of Epstein-Barr virus latent membrane protein 2 expression on the kinetics of early B cell infection. PLoS One 8: e54010, 2013.

16. Guasparri I, Bubman D and Cesarman E: EBV LMP2A affects LMP1-mediated NF-kappaB signaling and survival of lymphoma cells by regulating TRAF2 expression. Blood 111: 3813-3820, 2008.

17. Vrazo AC, Chauchard M, Raab-Traub N and Longnecker R: Epstein-Barr virus LMP2A reduces hyperactivation induced by LMP1 to restore normal B cell phenotype in transgenic mice. PLoS Pathog 8: e1002662, 2012.

18. Do NV, Ingemar E, Phi PT, Jenny A, Chinh TT, Zeng Y and Hu L: A major EBNA1 variant from Asian EBV isolates shows enhanced transcriptional activity compared to prototype B95.8. Virus Res 132: 15-24, 2008.

19. Chen YL, Liu CD, Cheng CP, Zhao B, Hsu HJ, Shen CL, Chiu SJ, Kieff E and Peng CW: Nucleolin is important for Epstein-Barr virus nuclear antigen 1-mediated episome binding, maintenance, and transcription. Proc Natl Acad Sci USA 111: 243-248, 2014.

20. Liu X, Tang J, Wang M, Ma Q and Wang Y: Visual detection and evaluation of latent and lytic gene expression during Epstein-Barr virus infection using one-step reverse transcription loop-mediated isothermal amplification. Int J Mol Sci 14: 23922-23940, 2013.

21. Daskalogianni C, Pyndiah S, Apcher S, Mazars A, Manoury B, Ammari N, Nylander K, Voisset C, Blondel M and Fåhraeus R: Epstein-Barr virus-encoded EBNA1 and ZEBRA: Targets for therapeutic strategies against EBV-carrying cancers. J Pathol 235: 334-341, 2015.

22. Pagès F, Galon J, Karaschuk G, Dudziak D, Camus M, Lazar V, Camilleri-Broët $S$, Lagorce-Pagès $C$, Lebel-Binay $S$, Laux $G$, et al: Epstein-Barr virus nuclear antigen 2 induces interleukin-18 receptor expression in B cells. Blood 105: 1632-1639, 2005.

23. Rowe M, Raithatha S and Shannon-Lowe C: Counteracting effects of cellular Notch and Epstein-Barr virus EBNA2: Implications for stromal effects on virus-host interactions. J Virol 88: 12065-12076, 2014.

24. Yue W, Gershburg E and Pagano JS: Hyperphosphorylation of EBNA2 by Epstein-Barr virus protein kinase suppresses transactivation of the LMP1 promoter. J Virol 79: 5880-5885, 2005.

25. Konforte D, Simard N and Paige CJ: Interleukin-21 regulates expression of key Epstein-Barr virus oncoproteins, EBNA2 and LMP1, in infected human B cells. Virology 374: 100-113, 2008. 
26. Young P, Anderton E, Paschos K, White R and Allday MJ: Epstein-Barr virus nuclear antigen (EBNA) 3A induces the expression of and interacts with a subset of chaperones and co-chaperones. J Gen Virol 89: 866-877, 2008.

27. Chen A, Zhao B, Kieff E, Aster JC and Wang F: EBNA-3B- and EBNA-3C-regulated cellular genes in Epstein-Barr virus-immortalized lymphoblastoid cell lines. J Virol 80: 10139-10150, 2006.

28. Tursiella ML, Bowman ER, Wanzeck KC, Throm RE,Liao J,Zhu J and Sample CE: Epstein-Barr virus nuclear antigen 3A promotes cellular proliferation by repression of the cyclin-dependent kinase inhibitor p21WAF1/CIP1. PLoS Pathog 10: e1004415, 2014.

29. Saha A and Robertson ES: Impact of EBV essential nuclear protein EBNA-3C on B-cell proliferation and apoptosis. Future Microbiol 8: 323-352, 2013.

30. Portal D, Zhou H, Zhao B, Kharchenko PV, Lowry E, Wong L, Quackenbush J, Holloway D, Jiang S, Lu Y, et al: Epstein-Barr virus nuclear antigen leader protein localizes to promoters and enhancers with cell transcription factors and EBNA2. Proc Natl Acad Sci USA 110: 18537-18542, 2013.
31. Portal D, Zhao B, Calderwood MA, Sommermann T, Johannsen E and Kieff E: EBV nuclear antigen EBNALP dismisses transcription repressors NCoR and RBPJ from enhancers and EBNA2 increases NCoR-deficient RBPJ DNA binding. Proc Natl Acad Sci USA 108: 7808-7813, 2011.

32. Yokoyama A, Tanaka M, Matsuda G, Kato K, Kanamori M, Kawasaki H, Hirano H, Kitabayashi I, Ohki M, Hirai K, et al: Identification of major phosphorylation sites of Epstein-Barr virus nuclear antigen leader protein (EBNA-LP): Ability of EBNA-LP to induce latent membrane protein 1 cooperatively with EBNA-2 is regulated by phosphorylation. J Virol 75: 5119-5128, 2001. 